\title{
Emergency surgery for a ruptured bronchial artery aneurysm: a case report
}

\author{
Tsutomu Koyama”, Shunichiro Matsuoka”, Takashi Eguchi^, Sachie Koike, Testu Takeda, \\ Kentaro Miura, Kazutoshi Hamanaka, Kimihiro Shimizu \\ Division of General Thoracic Surgery, Department of Surgery, Shinshu University School of Medicine, Matsumoto, Japan \\ \#These authors contributed equally to this work. \\ Correspondence to: Takashi Eguchi, MD, PhD, FCCP. Attending Surgeon/Senior Assistant Professor, Division of General Thoracic Surgery, \\ Department of Surgery, Shinshu University Hospital/Shinshu University School of Medicine, 3-1-1 Asahi, Matsumoto 390-8621, Japan. Email: \\ eguchi_t@shinshu-u.ac.jp.
}

Background: A mediastinal bronchial artery aneurysm is a rare disease, and its rupture causes mediastinal hematoma and/or hemothorax, which results in hemorrhagic shock. Radiological intervention is considered as a first-line treatment for both ruptured or unruptured mediastinal bronchial artery aneurysms because it is less invasive than surgical interventions. However, a hemodynamically unstable patient might require an emergency surgery. We herein report a case of an 86-year-old female patient who had a ruptured mediastinal bronchial artery aneurysm, which led to cardiac arrest, and who survived after an emergency thoracotomy carried out to achieve hemostasis.

Case Description: The patient presented with sudden-onset, severe back pain. A chest computed tomographic scan with contrast revealed a massive left hemothorax and mediastinal hematomas with extravasation. A three-dimensional reconstruction showed a saccular aneurism in the aorto-pulmonary window area, which was connected to the bronchial artery. The patient was urgently transferred to our hospital for transarterial embolization. However, due to the hemodynamic instability, we decided to perform an emergency thoracotomy to achieve hemostasis. Despite continuous volume replacement with blood transfusion and fluid with vasopressor infusion, she went into cardiac arrest during the induction of general anesthesia and was provided cardiac resuscitation. Under the guidance of three-dimensional imaging, an anterolateral incision was made on the left side of the chest just lateral to the level of the ruptured aneurysm based on the imaging, and the third intercostal space was opened. A saccular aneurism with arterial bleeding was identified in a mediastinal hematoma in the aortopulmonary window area just below the incision. The afferent and efferent arteries of the aneurysm were ligated to achieve hemostasis.

Conclusions: An important practice point is that although radiological interventions were not able to be performed due to patient's hemodynamic instability, emergency hemostasis was successfully achieved, with guidance from three-dimensional computed tomographic imaging despite massive hemothorax and hematoma.

Keywords: Bronchial artery aneurysm (BAA); rupture; three-dimensional computed tomography (3D-CT); hemothorax; case report

Received: 28 August 2020; Accepted: 24 January 2021; Published online: 07 February 2021.

doi: $10.21037 /$ ccts-20-145

View this article at: http://dx.doi.org/10.21037/ccts-20-145

\footnotetext{
^ ORCID: 0000-0001-9460-0696.
} 


\section{Introduction}

A bronchial artery aneurysm (BAA) is a rare disease. A BAA is categorized as either intrapulmonary or mediastinal, according to the site of aneurysm, and the symptoms of a ruptured BAA depend on its type. Rupture of a mediastinal BAA causes mediastinal hematoma and/or hemothorax, which results in hemorrhagic shock (1). Treatment options for BAAs include transarterial embolization (TAE), endovascular stent grafting for the aorta (thoracic endovascular aortic repair, TEVAR), and open surgical procedures. TAE has been widely considered as a first-line treatment for both ruptured or unruptured BAAs because it is less invasive than other methods (2). Recently, the number of reports of successful TEVAR for mediastinal BAA has been increasing $(3,4)$. Although rare, an open surgical procedure may be required in hemodynamically unstable patients, and therefore, general thoracic surgeons should be familiar with a clinical picture and a treatment strategy for a ruptured BAA.

We report the case of a patient who had a ruptured BAA, which led to cardiac arrest, and was rescued by an emergency thoracotomy for hemostasis. This is a unique and thought-provoking case for general thoracic surgeons because radiological interventions were not possible due to the patient's hemodynamically unstable status and an emergency hemostasis was successfully achieved with three-dimensional computed tomographic (3D-CT) image guidance. We present the following case in accordance with the CARE reporting checklist (available at https://ccts. amegroups.com/article/view/10.21037/ccts-20-145/rc).

\section{Case presentation}

An 86-year-old female patient who was a non-smoker but had a history of hypertension who had been taking cilostazol presented with sudden-onset, severe back pain without any prodromal signs or symptoms. A chest CT scan with contrast revealed a massive left hemothorax and mediastinal hematomas with extravasation of contrast medium from a saccular aneurism (Figure 1). A 3D reconstruction of the CT scan also showed a saccular aneurism in the aorto-pulmonary window area, which was connected to the bronchial artery, suggesting a ruptured BAA but excluded aortic dissection (Figure 2). The patient was urgently transferred to our hospital for TAE. However, she became hemodynamically unstable just after arrival in our emergency department. We initiated rapid volume replacement with Ringer solution

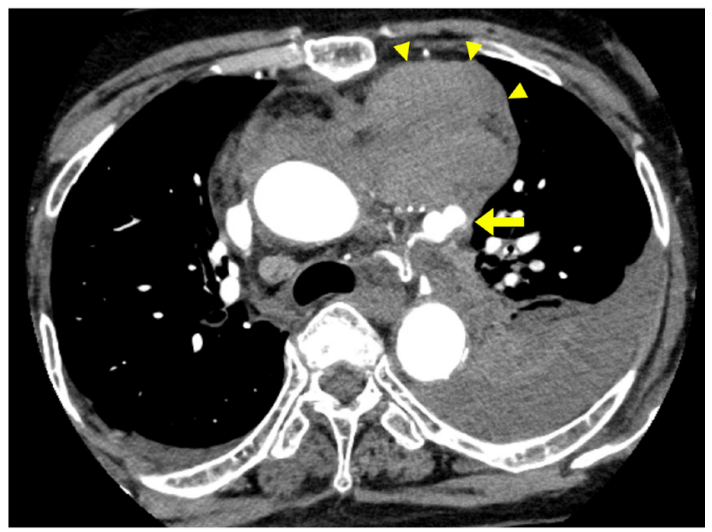

Figure 1 A preoperative computed tomography scan with contrast showing an aneurism with extravasation (arrow), hemothorax and intrathoracic hematomas (arrowheads).

and transfusion of warmed red blood cells, and a continuous vasopressor (norepinephrine) infusion through the peripheral veins. Due to her hemodynamic instability, we performed tracheal intubation in the emergency department to initiate mechanical ventilation and decided to perform an emergency thoracotomy to achieve hemostasis rather than TAE.

Immediately on arrival in the operating room, central venous access through the right jugular vein was created. In addition to the red blood cell transfusion, we initiated a transfusion of fresh frozen plasma in the operating room. Despite continuous volume replacement with vasopressor infusion, the patient suddenly went into cardiac arrest with pulseless electrical activity during induction of general anesthesia in the supine position. We immediately performed an external cardiac massage by chest compression for 2 minutes, which revived her. Subsequently, the patient was placed in the right lateral decubitus position.

A $15-\mathrm{cm}$ anterolateral incision was made in the left chest just lateral to the ruptured aneurism based on the preoperative $3 \mathrm{D}-\mathrm{CT}$, and the $3 \mathrm{rd}$ intercostal space was opened (Figure 2). A massive bloody effusion in the left pleural cavity drained out at the time of opening. A saccular aneurism with arterial bleeding was identified within mediastinal hematoma in the aortopulmonary window area just below the incision. The neck of the saccular aneurism was grasped by forceps to achieve temporary hemostasis (Figure 3). Subsequently, the afferent and efferent arteries of the aneurism were ligated, and the aneurism was resected (Figure 4). Bloody effusion and hematomas in the pleural cavity and mediastinum were evacuated and two chest tubes were placed. The operating time was 52 minutes 

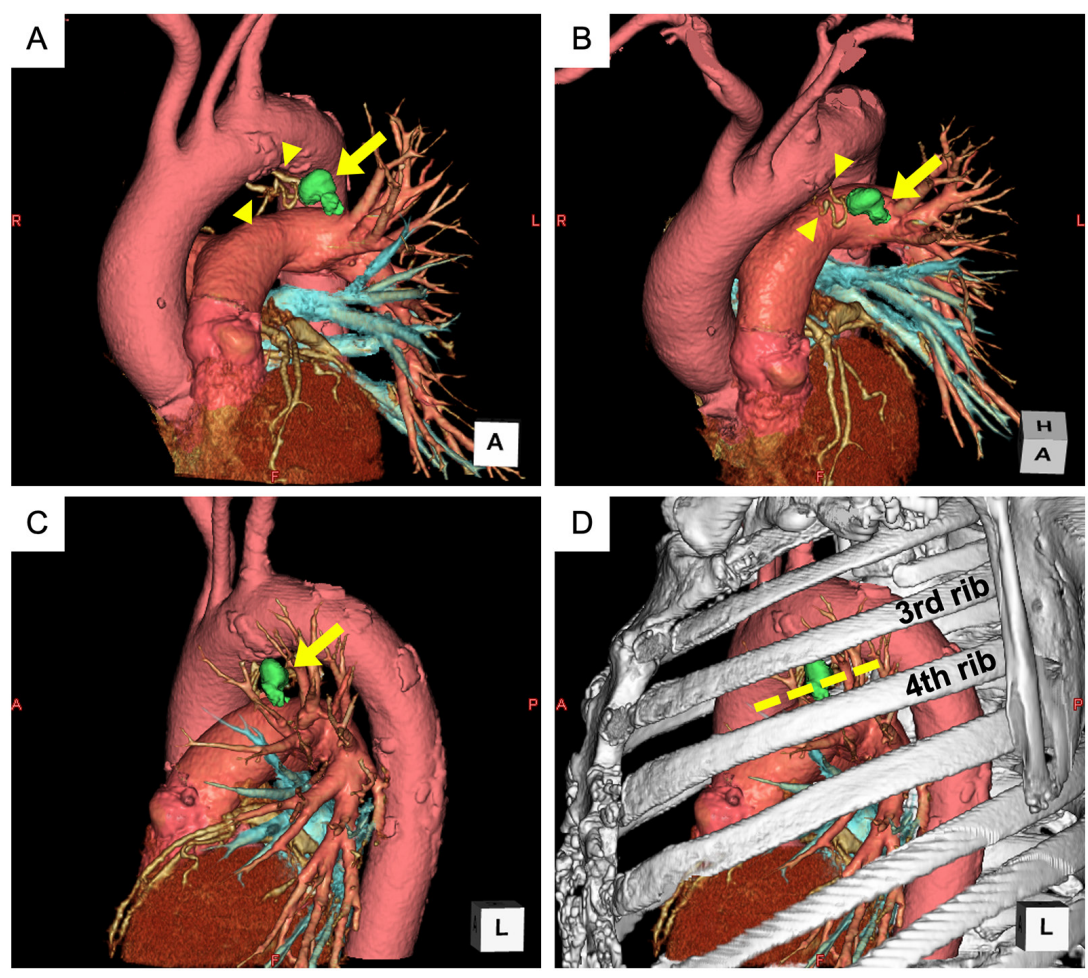

Figure 2 A preoperative three-dimensional computed tomography scan showing an aneurysm arising from the bronchial artery in the aortopulmonary area (arrows) with an afferent and efferent arteries (arrowheads). (A) Anterior view; (B) antero-cranial view; (C) lateral view; (D) lateral view with the ribs. The aneurism exists just under a planned incision on the third intercostal space (dotted line).

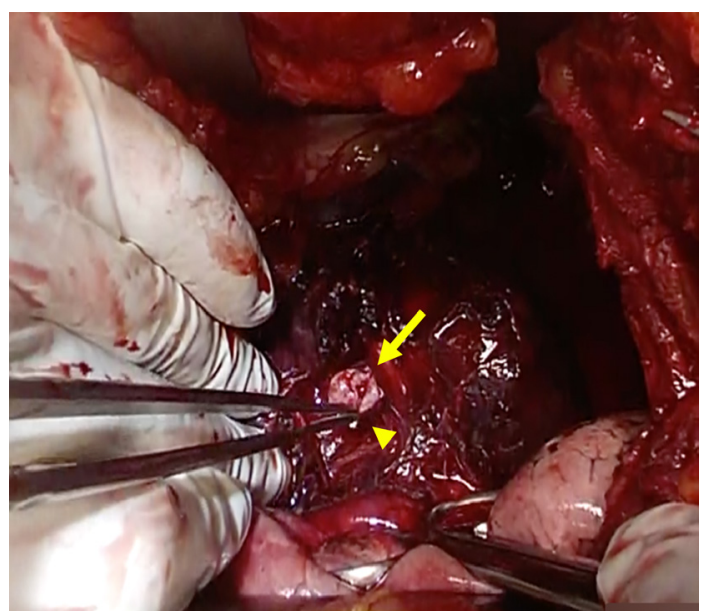

Figure 3 An intraoperative image showing a ruptured saccular aneurysm (arrow) that was identified just below a third intercostal thoracotomy incision. Temporary hemostasis was achieved by grasping the neck of the aneurysm using forceps (arrowhead). and estimated blood loss including pleural bloody effusion and hematoma was $4,300 \mathrm{~mL}$. Although the patient had a cardiac arrest before surgery, a CT scan of her head after surgery revealed no hypoxic encephalopathy. The patient was transferred to the intensive care unit with mechanical ventilation.

The postoperative course was complicated by intrathoracic bleeding from the left intercostal artery, which required further surgery to achieve hemostasis on day one after surgery. After that, her postoperative course was uneventful. She was extubated on day eight and transferred to an affiliated hospital for rehabilitation on day 15. At four months post-surgery, the patient was alive and had no symptoms.

All procedures performed in this study were in accordance with the ethical standards of the institutional and/or national research committee(s) and with the Helsinki 


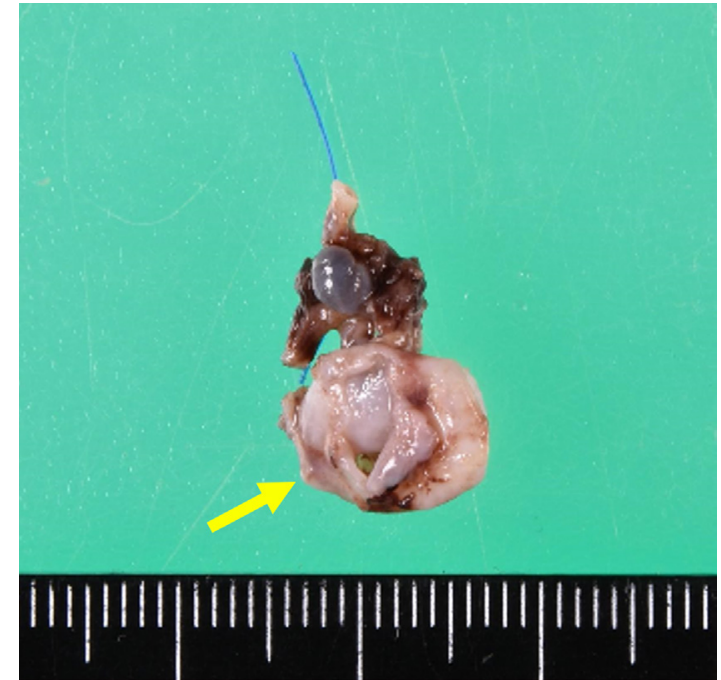

Figure 4 A resected bronchial artery aneurysm with a rupture in the apex of the aneurism (arrow).

Declaration (as revised in 2013). Written informed consent was obtained from the patient for publication of this case report and accompanying images. A copy of the written consent is available for review by the editorial office of this journal.

\section{Discussion}

BAA is a rare disease that is seen in fewer than $1 \%$ of patients selected for bronchial angiography (5). Due to recent increase of TAE, only a few cases of BAA are treated by surgery. However, a hemodynamically unstable patient might require an emergency surgery. In our case, the patient was initially transferred to our hospital for TAE. Due to her hemodynamic instability, we decided to perform an emergency thoracotomy. There are several case reports about successful treatment for a ruptured mediastinal BAA with TEVAR. Recently, Kikutani et al. reported a case of ruptured mediastinal BAA, which led to cardiopulmonary arrest (CPA) during the TAE procedure. The patient was revived by cardiopulmonary resuscitation with intra-aortic balloon occlusion, and after hemodynamic stabilization, the case was successfully treated with urgent TEVAR (3). This case reported by Kikutani et al. achieved temporal hemodynamic stability probably due to successful intraaortic balloon occlusion. In our case, an intravascular intervention using angiography was not available in the operating room when the patient went into cardiac arrest; hence, we resuscitated her by chest compression and performed an emergent thoracotomy to achieve hemostasis immediately after the resuscitation. However, an emergent TEVAR in the operating room with preparedness for thoracotomy in case of unsuccessful hemostasis could be an alternative option. In addition, given the potential lifethreatening implications of a BAA rupture, an intervention including TAE or TEVAR should be considered in cases where an unruptured BAA is discovered unexpectedly (6).

We have previously reported the advantages and usefulness of 3D-CT reconstruction for understanding individual anatomical findings during pulmonary segmentectomy $(7,8)$. In addition, 3D-CT has enabled a more precise understanding of the spatial relationship between anatomical structures. In our case, 3D-CT allowed better understanding of the shape and location of BAA, which helped us to determine the appropriate position of the thoracotomy (namely just above the ruptured BAA) and identify the ruptured BAA even when obscured by massive bloody effusion and hematoma.

\section{Conclusions}

We present the case of a patient with a ruptured BAA who was rescued by surgery. Significant findings of this case were that a ruptured BAA caused a massive hemothorax and eventually a CPA, but the patient was rescued with emergency surgery. We used 3D-CT scanning to identify the ruptured BAA during emergency surgery.

In conclusion, the rupture of a mediastinal BAA, although rare, may cause a life-threatening hemorrhage, so early intervention should be considered, and $3 \mathrm{D}$ CT scanning is useful to guide surgical treatment in an emergency situation.

\section{Acknowledgments}

We would like to thank Editage (www.editage.com) for English language editing.

Funding: None.

\section{Footnote}

Reporting Checklist: The authors have completed the CARE reporting checklist. Available at https://ccts.amegroups. com/article/view/10.21037/ccts-20-145/rc

Peer Review File: Available at https://ccts.amegroups.com/ article/view/10.21037/ccts-20-145/prf 
Conflicts of Interest: All authors have completed the ICMJE uniform disclosure form (available at https://ccts.amegroups. com/article/view/10.21037/ccts-20-145/coif). TE serves as an unpaid editorial board member of Current Challenges in Thoracic Surgery from February 2020 to January 2022. The other authors have no conflicts of interest to declare.

Ethical Statement: The authors are accountable for all aspects of the work in ensuring that questions related to the accuracy or integrity of any part of the work are appropriately investigated and resolved. All procedures performed in this study were in accordance with the ethical standards of the institutional and/or national research committee(s) and with the Helsinki Declaration (as revised in 2013). Written informed consent was obtained from the patient for publication of this case report and accompanying images. A copy of the written consent is available for review by the editorial office of this journal.

Open Access Statement: This is an Open Access article distributed in accordance with the Creative Commons Attribution-NonCommercial-NoDerivs 4.0 International License (CC BY-NC-ND 4.0), which permits the noncommercial replication and distribution of the article with the strict proviso that no changes or edits are made and the original work is properly cited (including links to both the formal publication through the relevant DOI and the license). See: https://creativecommons.org/licenses/by-nc-nd/4.0/.

\section{References}

1. Di X, Ji DH, Chen Y, et al. Endovascular treatment of

doi: $10.21037 /$ ccts-20-145

Cite this article as: Koyama T, Matsuoka S, Eguchi T, Koike S, Takeda T, Miura K, Hamanaka K, Shimizu K. Emergency surgery for a ruptured bronchial artery aneurysm: a case report. Curr Chall Thorac Surg 2023;5:11. ectopic bronchial artery aneurysm with brachiocephalic artery stent placement and coil embolization: A case report and literature review. Medicine (Baltimore) 2016;95:e4461.

2. Hou Z, Wang J, Peng D, et al. Massive hemoptysis due to multiple bronchial artery aneurysms and multiple aneurysmal dilations: A case report. Radiol Case Rep 2017;13:24-7.

3. Kikutani K, Itai J, Ota K, et al. A Ruptured Mediastinal Bronchial Artery Aneurysm Treated with Urgent Thoracic Endovascular Aortic Repair. Intern Med 2020;59:1283-6.

4. Matsumoto T, Uchida T, Ono T, et al. Bronchial Artery Aneurysm Treated Using Aortic Stent Graft Alone: A Case Report. Ann Vasc Dis 2017;10:152-4.

5. Fujita J, Akashi K, Kunikane H, et al. A case of bronchial artery aneurysm demonstrating a mass shadow on chest X-ray film. Nihon Kyobu Shikkan Gakkai Zasshi 1991;29:1591-5.

6. Kalangos A, Khatchatourian G, Panos A, et al. Ruptured mediastinal bronchial artery aneurysm: a dilemma of diagnosis and therapeutic approach. J Thorac Cardiovasc Surg 1997;114:853-6.

7. Shimizu K, Nakazawa S, Nagashima T, et al. 3DCT anatomy for VATS segmentectomy. J Vis Surg 2017;3:88.

8. Eguchi T, Takasuna K, Kitazawa A, et al. Threedimensional imaging navigation during a lung segmentectomy using an iPad. Eur J Cardiothorac Surg 2012;41:893-7. 